\title{
Paediatric multiple sclerosis: A case report of missed and dismissed diagnosis
}

\author{
DOI: https://doi.org/10.32007/jfacmedbagdad.6311776
}

\author{
Nebal W. Saadi* FICP, CABP, FIBPN \\ Qusay A. Fahad** FIBMS
}

@( $) \Theta$ This work is licensed under a Creative Commons Attribution-NonCommercial 4.0 International License.

Abstract:

Background: Multiple Sclerosis is a chronic inflammatory autoimmune disease of the central nervous system. Up to $10 \%$ of Multiple Sclerosis patients have onset in paediatric age group. Although the clinical profile of Multiple Sclerosis appears similar to that seen in adults, several features may differ and specific issues arise in children.

J Fac Med Baghdad 2021; Vol.63, No. 1 Received: March 2021 Accepted: April, 2021 Published: May, 2021
Case report: Here, we report a 12-year-old girl who presented with a 3-year history of episodes of seizures, facial nerve palsy and finally fatigue and cognitive impairment were noted and interfered with her academic performance. Because of the presumed atypical clinical presentations, the diagnosis was missed then dismissed despite neuro-imaging features and CSF immunological findings that were highly suggestive of Multiple Sclerosis. Later on, evolution of the disease by neuro-imaging helped confirm the diagnosis and direct toward the delayed therapy.

Conclusion: Age-related variations in presentations of Multiple Sclerosis need to be considered. Maintaining a high index of suspicion speeds up diagnosis and use of appropriate treatment and thus minimizing associated morbidities.

Keywords: Multiple sclerosis, MRI, Paediatric
Introduction:

Multiple sclerosis (MS) is a chronic immunemediated inflammatory disorder of the central nervous system (CNS) that results in demyelination, gliosis and axonal damage. Most common age of onset is between 20 and 40 years, with the 30 years is the peak age of incidence $(1,2)$. Geographic factors govern its prevalence as it was reported to range from 1.35 to 2.5 per 100.000 in United State to over 248 per 100.000 in western Canada $(3,4)$. Appearance of MS before the age of 16 years (and sometimes before the age of 18 years) defines paediatric MS. It can be first manifested before age of 18 years in $3-10 \%$ of all cases (5). Therefore, physicians should remain alert to this possible diagnosis in children patients. In children, it mostly presents at age of 12 to 13 years $(4,6)$. Multifocal presentation is commonly reported in children before puberty, in contrast to the monofocal manifestations in adolescents (7). In children, brainstem and cerebellar manifestations are more frequently present at onset than spinal cord symptoms, as compared to adults (8). Relapsingremitting course is manifested at a higher frequency $(98 \%)$ in paediatric MS patients as compared with adult patients (84\%) (4). generally, differentials like systemic lupus erythematosus (SLE), neurosarcoidosis, Sjögren syndrome, leukodystrophies, hereditary metabolic disorders, and

* Department of Paediatric Neurology, College of Medicine, University of Baghdad. Correspondence Email: nebalpedneu2013@gmail.com.

** Department of Radiology, Baghdad Teaching Hospital, Medical City Complex, Email: Altalib74@yahoo.com. encephalitic or meningo-encephalitic infectious aetiologies, need to be considered and investigated before making a diagnosis of MS, particularly in atypical cases and younger children, where a variety of clinical presentations is encountered (9). Neuroplasticity in children may slow down the progression of the disease, however, moderate-tosevere disability is reached at a younger age (10). Dissemination of inflammatory demyelinating lesions in both space and time is a mandatory requisite to diagnose MS in children as well as in adults (9). Starting early treatment for paediatric MS is recommended by guidelines, and diseasemodifying therapies (DMTs) for adult patients with MS can also be applied in paediatric MS $(11,12)$.

\section{Case report:}

On 2015, a previously healthy 12-year-old girl, presented complaining of two generalized tonicclonic seizures, accompanied by fever. Her brain CT was normal. She was placed on Sodium Valproate and became free of symptoms. On 2017, she had sudden facial palsy, brain MRI was requested (Figure A) and Bell's palsy was diagnosed. She was treated with oral steroids for two weeks and recovered. On August, 2018 she had brief generalized tonic-clonic seizures and Sodium Valproate was re-started by a physician. She had decline in cognitive status with increasing fatigability, both of which, made her school experience challenging. She failed in the year proceeded her presentation, obtained lower marks and showed impairment of executive functions. Poor social responsiveness, reduced motor activity and numbness of the right leg were also documented. She 
presented on December 2018 and her examination showed an alert child, oriented with no dysmorphology or cutaneous marks. Her general and systemic physical examinations were normal. Neurological examination showed normal cranial nerves including fundi, normal motor functions apart from brisk biceps jerks, and normal sensory as well as cerebellar functions. New brain MRI (Figure B) revealed multiple white matter high-signal intensity foci on T2-weighted/FLAIR images. The lesions were disseminated in the supra- and infra-tentorial regions of the brain (periventricular, subcortical and cerebellum). They were specific for demyelination and highly suggestive of MS. Cerebrospinal fluid (CSF) showed normal general profile, elevated IgG index and oligoclonal bands were detected. Serum was analysed for systemic inflammatory diseases (ANA, ENA, ANCA, RF, aCL, IgG, IgM), showing normal results including those for Behcet's disease and thyroid hormones. Lupus anticoagulants test was elevated and became negative upon repetition. Visual-evoked potential was normal. She received pulsed 3-day intravenous Methylprednisolone, followed by 2 -week oral prednisolone. Partial improvement of cognitive and motor function was noted. She couldn't be enrolled in the Disease Modifying Drugs (DMDs) program as her scenario was questioned because of the presumed atypical presentations of MS. She was re-investigated for vasculitis and other mimics. Tests' results were negative, yet MS diagnosis was declined. Four months later, CNS neuro-imaging (Figure C) showed T1-hypointense lesion with ring/homogenous pattern of enhancement. The issue of signing up for the DMDs program of MS was re-negotiated. Finally, she received interferon and clinical improvement was continually reported. The latest revision of her signs and symptoms, after 18 months of receiving maintenance treatment, revealed no more fatigability, seizures, blurred vision and sensory symptoms. Her school performance was upgraded as documented by her teachers, parents and school marks. She reported symptoms of flu-like illness and sourness at the injection site. No update of her brain radiological status was obtained.
2017

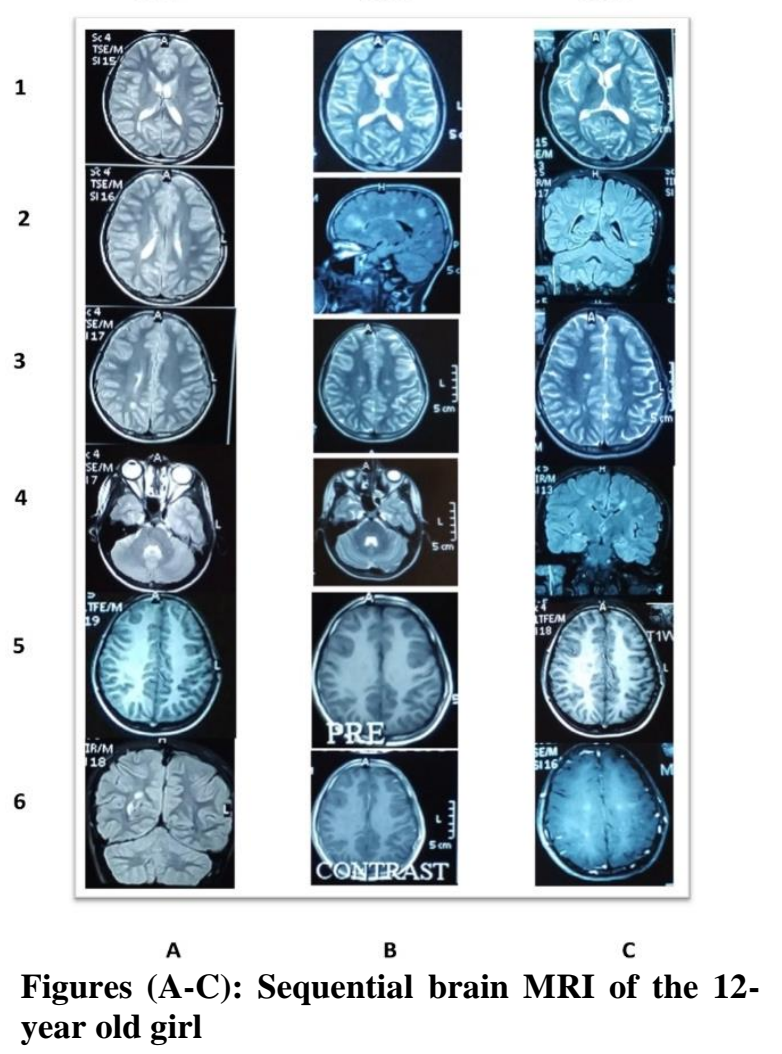

A: On 2017, discrete hyperintense foci in T2 WI, White Matter (WM) axial left parietal \& right frontal (2), axial right occipital and periventricular $(1,3)$ and Coronal FLAIR, periventricular (6). No lesion is seen in T1 WI (5) or cerebellum, T2-axial (4).

B: On December 2018, increase in the number of hyperintense foci in T2 and FLAIR, deep WM, axial, subcortical (1) \& sagittal (2), periventricular (3), right cerebellar lesion (4) and faint hypointense lesions in T1 WI in the deep WM on the right side (5) that wasn't enhanced (6).

C: On June 2019, relative reduction in the size and number of the hyperintense foci (1), yet it can be seen distributed in the WM, subcortical, deep and juxtacortical $(2,3,4)$ and increased hyperintense- signalling of two foci in the deep WM, on both sides that show ring/ homogenous enhancement.

\section{Discussion:}

In the present vignette, the initial presentations as discrete, brief and non-overlapped clinical events like seizures and facial palsy were not investigated appropriately and were treated as more benign entities despite the highly suspicious MRI features, which explains the initial missing in her diagnosis and is attributed to low awareness of physicians of the demyelinating disorders in children. Later on, MS diagnosis was dismissed by the neurology physicians, arguing the atypical presentations; seizures, craniopathy, cognitive decline and the positive lupus anticoagulant test. The prevalence rate of epilepsy in MS was estimated to be $2.3 \%$, with a risk of occurrence 3-6 times higher than that in general adult population. It can occur early in the course of MS. Inflammation and demyelination of the cortex and juxtacortical white matter can be possible pathological mechanisms (13, 14, and 15). In our patient, the load of brain lesions in the peri-temporal horn and lobe might explain the persistent seizure symptoms. Isolated cranial neuropathies rarely occur in multiple sclerosis, reported in $1.6 \%$ of all patients. 
Peripheral facial nerve palsy has an incidence of around $0.2 \%$, which could be due to an injury at the level of ipsilateral facial nucleus or pontine part of facial nerve $(16,17)$. Cognitive deficits $(\mathrm{CD})$ were found out in up to two thirds of paediatric MS. Manifestations like attention problems, impairment of language, impairment of information processing speed, fatigue, depression and anxiety are commonly observed, negatively impacting the quality of life and restricting the academic, social and entertainment activities (5,18). Displaying the radiological dissemination in space and time and eliminating the other neurological conditions are essential for the diagnosis of MS. Both of which were achieved in our case (Figures A-C). In the present scenario, the pattern of distribution of CNS lesions, the positive banding and elevated IgG index in CSF, the long duration of recurrent symptoms, the absence of other features supporting the diagnosis of vasculitis and other inflammatory non-demyelinating disorders, and the previously normal developmental profile, all favoured the diagnosis of MS. This case highlighted the distinctive features and clinical course of paediatric MS that are different from adult MS, which resulted in under-diagnosis and under-treatment. Age-related variations in presentations of MS need to be considered. Maintaining a high index of suspicion, speeds up diagnosis and use of appropriate treatment and thus minimizing associated morbidities. Closer collaboration between paediatric and adult neurology centres would promote sharing knowledge and conducting long-term studies in demyelinating disorders to improve the care of these children/ adolescents and their families.

\section{Authors' contribution:}

Nebal Waill Saadi: Conceptualization of and designing the study, analysis and interpretation of the data and drafting the manuscript for intellectual content

Qusay Abed Fahad: Analysis and interpretation of data.

\section{Acknowledgements}

We are grateful to the patient and her family for their informed consent and permission for publishing this report. We specially thank Dr Samaher Abdul Razaq (Oncology unit, Children Welfare Teaching Hospital) for her critical review of the manuscript.

Study funding: None

Author disclosures:

Nebal Waill Saadi reports no disclosures

Qusay Abed Fahad reports no disclosures

\section{References:}

1. Lucchinetti C, Bruck W, Parisi J, Scheithauer B, Rodriguez M, Lassmann H. Heterogeneity of multiple sclerosis lesions: implications for the pathogenesis of demyelination. Ann Neurol. 2000; 47: 707-17.
2. Hirofumi Ochi. Clinical features, diagnosis and therapeutic strategies in pediatric multiple sclerosis. Clinical and Experimental Neuroimmunology. 2017; 8(Suppl. 1): 33-39.

3. Banwell BL. Pediatric multiple sclerosis. Curr Neurol Neurosci Rep. 2004 May; 4(3): 245-52.

4. Renoux C, Vukusic S, Mikaeloff Y, Edan G, Clanet $M$, Dubois B, et al. Natural history of multiple sclerosis with childhood onset. N Engl J Med. 2007 Jun; 356(25): 2603-13.

5. Ghezzi A, Baroncini D, Zaffaroni M, Comi G. Paediatric versus adult MS: similar or different?. Multiple Sclerosis and Demyelinating Disorders. 2017; 2(5): 2.

6. Yavari MJ, Inaloo S, Saboori S. Multiple sclerosis in children: A review of clinical and paraclinical features in 26 cases. Iran J Child Neurol. 2008; 2(4): 41-46.

7. Banwell B, Ghezzi A, Bar-Or A, Mikaeloff Y, Tardieu M. Multiple sclerosis in children: clinical diagnosis, therapeutic strategies, and future directions. Lancet Neurol. 2007; 6: 887-902.

8. Chabas D, Strober J, Waubant E. Pediatric multiple sclerosis. Curr Neurol Neurosci Rep. 2008; 8: 434-41.

9. Banwell B, Kennedy J, Sadovnick D, et al. Incidence of acquired demyelination of the CNS in Canadian children. Neurology. 2009; 72: 232-9.

10. Raed Alroughaniland Alexey Boyko. Pediatric multiple sclerosis: a review. BMC Neurology. 2018; 18: 27.

11. Pohl D, Waubant E, Banwell B, et al. Treatment of pediatric multiple sclerosis and variants. Neurology. 2007; 68(Suppl 2): S54-65.

12. Ghezzi A, Banwell B, Boyko A, et al. The management of multiple sclerosis in children: a European view. Mult Scler. 2010; 16(10): 1258-67.

13. Kelley BJ, Rodriguez M. Seizures in Patients with Multiple Sclerosis: Epidemiology, Pathophysiology and Management. CNS Drugs. 2009 Oct; 23(10): 805-15.

14. Koch M, Uyttenboogaart M, Polman S, De Keyser J. Seizures in multiple sclerosis. Epilepsia. 2008 Jan; 49(6): 948-53.

15.Zeqiraj K, Shatri N, Kruja J, Blyta A, Isaku E, Dakaj N. Epileptic Seizures in Multiple Sclerosis Patients. Turk J Neurol. 2013; 19(2).

16. Saleh $C$, Patsi O, Mataigne F, Beyenburga $S$. Peripheral (Seventh) Nerve Palsy and Multiple Sclerosis: A Diagnostic Dilemma - A Case Report. Case Rep Neurol. 2016 Jan23; 8(1): 27-33.

17. Abkur TM. Isolated peripheral facial nerve palsy in multiple sclerosis. BMJ Case Rep. 2018 Nov 28; 11(1).

18. Wingerchuk DM. Multiple Sclerosis and Other CNS Inflammatory Diseases. Continuum Jun 2019 25; 3: 800 . 


\title{
التصلب المتعدد لاى الأطفال : تقرير عن حالة مع تثخيص مفتقد و مرفوض
}

\author{
د. نبال وائل سعدي \\ د. قصي عبد فهر
}

الخلاصة

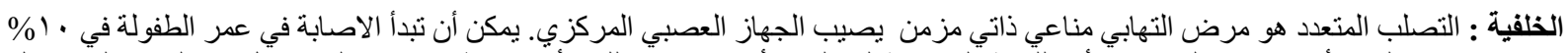

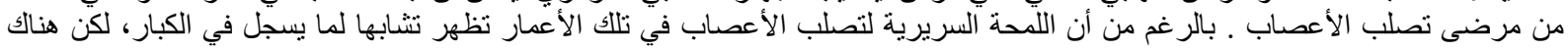

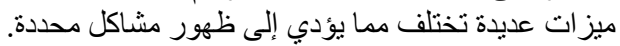

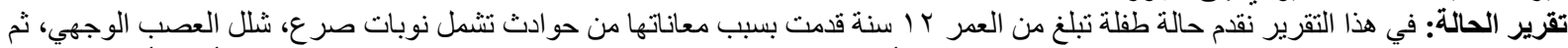

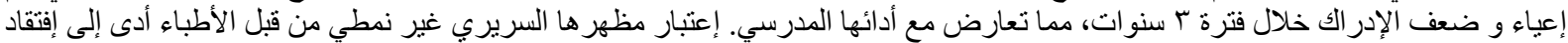

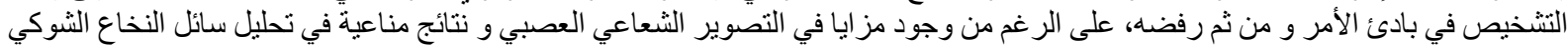

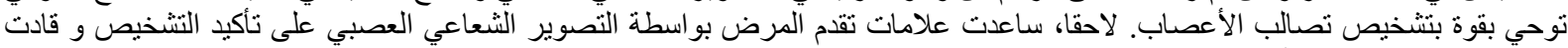

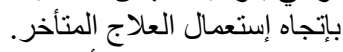
الإستنتاج: لا بد من الأخذ بعائن الإعنبار التغييرات ذات الصلة بالعمر الخاصة بمظاهر تصلب الأعصاب. المحافظة على مؤشر شك مرتفع بسرع

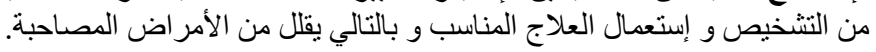

